\title{
Política educacional para una modalidad e-learning en la universidad a partir de la pandemia
}

\author{
Sindy Diamela Tippe Marmolejo*1; Susan Lizeth Soto Giraldo ${ }^{2}$ \\ ${ }^{1}$ https://orcid.org/0000-0002-5175-5214, Universidad Peruana de Ciencias Aplicadas, Lima, Perú. ${ }^{2}$ https://orcid.org/0000-0002- \\ 6081-4597. Universidad Nacional de Educación Enrique Guzmán y Valle, Lima, Perú
}

Citar como: Tippe, S., \& Soto, S. (2021). Política educacional para una modalidade-learning en la universidad a partir dela pandemia. Revista Digital de Investigación en Docencia Universitaria, 15(2), e1306. https://doi.org/10.19083/10.19083/ridu.2021.1306

Recibido: 15/09/2020. Revisado: 20/09/2020. Aceptado: 03/06/2021. Publicado: 26/11/2021.

\begin{abstract}
Resumen
Introducción: En este artículo, basándose en la exploración teórica, se ha identificado la necesidad de plantear propuestas hacia la construcción de una política educacional de mínimos necesarios para la modalidad e-learning en la universidad en el contexto de pandemia por la Covid-19. Antecedentes: En consecuencia, se propuso como método el heurístico y el hermenéutico, a partir de los cuales se desprende que, la virtualización de la educación peruana ha significado un proceso acelerado y no planificado, a partir del cual la metodología aplicada en la enseñanza en línea ha evidenciado grandes similitudes respecto de la educación presencial. Discusión: Es preciso establecer nuevas condiciones básicas de calidad del servicio educativo e-learning en el nivel superior universitario, que permitan a las instituciones universitarias adaptarse tanto a las exigencias del nuevo mercado educativo como a los perfiles de sus potenciales estudiantes. Asimismo, por su parte, cada gobierno de turno debe desarrollar las gestiones pertinentes para generar condiciones óptimas que ayuden a reducir las brechas de acceso y aquella relacionada con las competencias digitales.
\end{abstract}

Palabras clave: e-learning; competencias digitales; enseñanza superior; educación a distancia; política educacional.

\section{Educational policies for e-learning teaching in universities after the pandemic}

\begin{abstract}
Introduction: In this article, based on the theoretical exploration, the need to propose proposals towards the construction of an educational policy of the minimum necessary for the e-learning modality in the university has been identified in the context of the Covid-19 pandemic. Background: Consequently, the heuristic and hermeneutical method were proposed, from which it follows that the virtualization of Peruvian education has meant an accelerated and unplanned process, from which the methodology applied in online teaching has evidenced great similarities with respect to face-to-face education. Discussion: It is necessary to establish new basic conditions for the quality of the e-learning educational service at the higher university level, which allow university institutions to adapt both to the demands of the new educational market and to the profiles of their potential students. Likewise, for its part, each government in turn must develop the pertinent steps to generate optimal conditions that help reduce access gaps and that related to digital skills.

Keywords: e-learning; digital skills; higher education; distance education; educational policy.
\end{abstract}

\section{${ }^{*}$ Correspondencia:}

Sindy Diamela Tippe Marmolejo, druzila28@gmail.com

Susan Soto Giraldo, susan.soto.giraldo@gmail.com 


\section{Introducción}

Finalizó el primer semestre del año 2020 y nuevos retos se generaron a raíz de una pandemia sin precedentes. Muchos países del mundo entraron en cuarentena $\mathrm{y}$, consecuentemente, se paralizaron actividades de toda índole. Además, hasta ahora, no se calcula cuál será el impacto generado en los diversos sectores de la sociedad, entre ellos, el de educación. Sin embargo, en este rubro no es difícil predecir que, debido a la desaceleración económica, los recortes presupuestales se harán visibles en el futuro, así como el aumento de la deserción educativa; pero esta crisis, sin duda, puede ser vista también como una posibilidad de transformación de la prestación de servicios (Figallo et al., 2020), a partir del cual la educación virtual sea una alternativa viable en la educación del siglo XXI (Organización de las Naciones Unidas para la Educación, la Ciencia y la Cultura[Unesco], 2020b).

La cuarentena trajo consigo el cierre de centros de educación escolar y de universidades e instituciones de educación superior como medida de contingencia para frenar, de alguna manera, el avance del brote infeccioso. En ese sentido, un reporte de la Unesco (2020b) expone que, en marzo de 2020, 184 países habían cerrado sus instalaciones educativas, lo cual significó la afectación de 1500 millones de estudiantes aproximadamente; esto sumado a cuantiosas pérdidas económicas. Asimismo, dicha situación, en el contexto de las casas de estudios universitarios, encuentra un remoto antecedente en la epidemia causada por la peste negra, en Inglaterra, la cual ocasionó el cierre temporal de la Universidad de Cambridge en 1665 (Buendía, 2020).

Por otro lado, en el Perú, la realidad universitaria es similar a la de otros países, pues se calcula que alrededor de 650 mil estudiantes peruanos dejarán sus estudios: 350 mil universitarios y 300 mil estudiantes de institutos (Figallo et al., 2020). Sin embargo, en este contexto retador, las tecnologías de la información y la comunicación (TIC) emergen, otorgándole protagonismo a la educación a distancia en modalidad virtual; lo cual se traduce como la única respuesta a la situación provocada por la COVID-19 (Gortazar et al., 2020; Pedró, 2020). Al respecto, el 15 de mayo de 2020, ya se habían registrado 59 universidades licenciadas (26 públicas y 33 privadas) para la continuidad del servicio educativo a través de la enseñanza virtual (Figallo et al., 2020).

Sin embargo, el contexto de pandemia ha conducido la incipiente virtualización de la educación peruana a un proceso acelerado y no planificado. Ello ha implicado que se reflexione acerca de la metodología aplicada en la enseñanza en línea, la cual debe evidenciar claras diferencias respecto de la educación presencial.

Asimismo, no se debe dejar de considerar las condiciones bajo las cuales se está llevando a cabo este proceso, pues la modalidad virtual aplicada en un contexto de emergencia no implica, en sí misma, su ejecución a cabalidad ni un verdadero cambio en el modelo institucional (García-Peñalvo, 2020b). En consecuencia, la adopción de esta medida de emergencia, en un contexto ya existente de disparidad de acceso tecnológico y competencias digitales, puede aumentar la brecha de las desigualdades en la educación (Unesco, 2020a).

Ante ello, el objetivo de este artículo es contribuir con la exploración de uno de los campos que ha tomado protagonismo en este contexto de emergencia sanitaria: la búsqueda de un modelo educacional de la enseñanza en línea, lo cual implica el planteamiento de políticas que velen por una enseñanza de calidad brindada a través de la modalidad e-learning. Para ello, se ha utilizado como marco de referencia la revisión de literatura relevante concerniente a los últimos 10 años.

\section{Modalidad E-learning en la Universidad}

La educación no presencial y su relación con las Tecnologías de la Información y Comunicación han introducido una serie de términos y conceptos que son de suma importancia manejar para su diferenciación.

Al respecto, Huertas et al., (2018), sugieren tomar en cuenta terminología relevante al respecto: 1) Cursos a distancia: se trata de cursos que no se imparten en el campus universitario y que requieren de Internet o material impreso para su realización; 2) Cursos online: son cursos que requieren de Internet como mecanismo principal para llevar a cabo las actividades pedagógicas que 
pueden ser síncronos y asíncronos; 3) Programas online: son programas acreditados e impartidos de manera online; 4) Cursos semipresenciales: el diseño de estos combina la enseñanza online con la presencial, también se les denomina híbridos, mixtos o blended.

En cuanto al término e-learning, este es utilizado para denominar a la educación online y se refiere a aquella tendencia en la que la enseñanza y el aprendizaje se efectúan a través de medios electrónicos (García-Peñalvo, 2020b; Mora-Vicarioli, 2019). Asimismo, Cevallos y Caraguay (2013) la definen como aquella modalidad que usa redes de ordenadores, recursos informáticos y de telecomunicaciones para llevar a cabo el proceso de enseñanza y aprendizaje de un curso o programa formativo para estudiantes que están geográficamente dispersos o que interactúan en tiempos diferidos del docente.

Una de las bondades de esta modalidad es que favorece al desarrollo de las habilidades blandas (García Peñalvo, 2020b; Mora-Vicarioli, 2019). Entre estas se hallan las de comunicación, trabajo en equipo, aprendizaje autónomo, pensamiento disruptivo, etc.; sin embargo, su uso y desarrollo depende de la mediación y estrategias que emplee el docente (Mora-Vicarioli, 2019). Dichas habilidades preparan a los estudiantes para la empleabilidad e incrementan la productividad en las empresas, y, en consecuencia, elevan el estándar de vida (Tito \& Serrano, 2016).

La expansión de la modalidad e-learning, en el sector educativo, se sigue desarrollando desde hace más de diez años aproximadamente, aunque es difícil precisar a partir de cuándo, específicamente, esta cobró presencia en el sector de la educación superior. Es posible afirmar que, a partir de la implementación de las TIC y la globalización, esta fue introduciéndose de forma discreta, inicialmente, en el sector privado. En Latinoamérica, la oferta varía de región en región; aunque aún no se evidencia alguna experticia consolidada; por el contrario, se observa un avance incipiente y desigual en dicha modalidad. Precisamente, al respecto, la escasa oferta es uno de los factores por el que, en el contexto de pandemia, no se han generado buenos resultados en la educación superior (Pedró, 2020).
En el caso del Perú, esta modalidad se ha venido introduciendo de manera muy reciente y solo en algunas casas de estudio superior. A diferencia de la educación básica regular del sector público, varias universidades e institutos implementaron, desde hace casi más de cinco años, dichas plataformas digitales como recursos importantes para el aprendizaje de sus estudiantes, aunque no fue la mayoría: "De hecho, no todas las universidades han tenido experiencias previas con cursos virtuales, al menos un $70 \%$ de ellas no lo había implementado nunca." (Figallo et al., 2020, p. 26).

En el país, estas instituciones han puesto en marcha el proceso de migración a la virtualidad de cierto porcentaje de su malla curricular, según lo permitido por la Superintendencia Nacional de Educación Superior Universitaria. Al respecto, en el artículo 47 de la Nueva Ley Universitaria N - 30220, se menciona que, las universidades pueden ofertar programas educativos a distancia a partir de entornos virtuales sujetos a las mismas condiciones de calidad que los programas presenciales; además, se precisa que "Los estudios de pregrado de educación a distancia no pueden superar el 50\% de créditos del total de la carrera bajo esta modalidad; y en caso de los estudios de maestría y doctorado, no podrán ser dictados exclusivamente bajo esta modalidad." (Ley $\mathrm{N}^{\circ}$ 30220, 2014).

El artículo mencionado fue modificado, a partir del contexto de pandemia, por una serie de normativas. Una de ellas fue la Resolución del Consejo Directivo No 039-2020-SUNEDU-CD (2020), la cual permite la educación a distancia sin ninguna restricción. Asimismo, en mayo del 2020, a través del Decreto Legislativo $N^{\circ} 1496$ se establecieron tres modalidades de estudio universitario: presencial, semipresencial y a distancia o no presencial, las cuales tienen por finalidad la ampliación del acceso a una educación de calidad y obedecen a las nuevas necesidades educativas de los estudiantes.

Por otro lado, la transición gradual o la implementación de la virtualidad, en algunas casas de estudio superior, no implicó que las competencias de sus docentes también se hayan desarrollado con la misma velocidad o en paralelo. Este aún es un proceso que la pandemia frenó a mitad de 
camino. Lo mismo ha sucedido con la aceptación de dicha modalidad por parte de los agentes educativos implicados y aún se cuestiona su eficacia, en contraposición con la modalidad presencial (Figallo et al., 2020).

El contexto de la pandemia no ha detenido dicho cuestionamiento. Por el contrario, ha generado mucha más desconfianza y reacciones negativas tanto por parte de los estudiantes como de sus apoderados. Entre sus principales argumentos sostienen que "la educación en línea no ofrece lo mismo que la presencial, que sus costos son inferiores, que las reducciones en las tasas de matrícula no son suficientes (...)." (Figallo et al., 2020, p. 24).

\section{Política Educacional Para una Modalidad E-learning de Calidad}

El impacto generado, en el sector de la educación superior, por el cambio de metodologías en la prestación del servicio, para garantizar la continuidad de este, está todavía en evaluación. Este cambio de metodología recae inevitablemente en dos aspectos de suma importancia, aunque hay otros más de diferente índole, estos son la calidad y la equidad (Pedró, 2020). A partir de la pandemia, se han develado, con mayor evidencia, las carencias y desigualdades en cuanto a dichos aspectos se refiere.

Al respecto, Buendía (2020) afirma que tanto en países de América Latina y el Caribe como en África se observan problemáticas que en corto tiempo se verán intensificadas: "Crecimiento sin calidad, inequidades en el acceso y en los logros de aprendizaje, y pérdida progresiva de financiamiento público, (...)." (Buendía, 2020, p. 27).

Por su parte, Fernández (2020) en Una pandemia imprevisible ha traído la brecha previsible, menciona tres brechas, cuya existencia datan de mucho antes de la pandemia, pero que, sin duda, se volvieron escandalosamente evidentes con esta. Una de ellas la denomina brecha de primer orden, se refiere al acceso a la tecnología, es decir, a los ordenadores o al Internet. Así también, se refiere a la dificultad en el acceso a materiales de aprendizaje y programas educativos online (Gortazar et al., 2020).

En cuanto a la brecha de segundo orden, se refiere a la disparidad en el uso de Internet; es decir, en la utilidad que adquiere Internet para cada tipo de familia. Gortazar et al. (2020) complementan ello, haciendo referencia al tiempo y la calidad del uso de Internet. Sobre esto, se afirma que, las familias con mayor nivel educativo e ingreso económico tienen mayor control sobre lo que hacen sus hijos frente a las pantallas y disponen de un bagaje más amplio de alternativas para sus momentos de ocio (Fernández, 2020).

Por último, la tercera brecha se refiere a las competencias digitales docentes. Tanto Fernández (2020) como García-Peñalvo (2020a) coinciden en señalar la brecha competencial, la cual se refiere a la carencia de competencias digitales, que se hacen más evidentes en el ámbito educativo público. Acerca del sector universitario, García-Peñalvo (2020a) afirma que, el profesorado expone una resistencia al cambio y que las casas de estudio carecen de estrategias que marquen el camino a seguir en la transformación digital.

Del mismo modo, Pedró (2020) sostiene tres razones directamente relacionadas con las brechas planteadas por Fernández y García. Estas son la desigualdad en cuanto al acceso a la tecnología, la insuficiente oferta de educación superior a distancia prepandemia y las competencias docentes y discentes respecto de la modalidad en mención. A partir de estas, avizora un desenlace no tan grato como resultado de las medidas de emergencia asumidas para la continuidad de las actividades pedagógicas en el sector de la educación superior.

En el contexto peruano, dichas brechas se observan con sus propias particularidades del siguiente modo: con respecto a la brecha del uso, desde hace más de diez años el empleo de Internet es visto como una actividad necesaria, complementaria e importante para el estudiante. Al respecto, la población que usa Internet a diario lo emplea, mayormente, en comunicación; en segundo lugar, en actividades de entretenimiento (juegos de video, música o películas); $y$, en tercer lugar, para obtener información (Instituto Nacional de Estadística e Informática [Inei], 2020). Por otro lado, de los hogares que poseen una computadora, el 94\% lo emplean en actividades académicas, el 5.7\% lo comparte entre las anteriores y el trabajo y el $0.4 \%$ lo emplea en actividades laborales (Inei, 2020). 
Es importante señalar que, en el estudio anterior, se identificó una diferenciación de uso según género, a partir de la cual el empleo del Internet lo encabeza la población masculina. Además, otro segmento importante de usuarios son los jóvenes y adolescentes.

Por otro lado, en cuanto a la brecha de acceso, hasta junio de 2020, de 100 hogares peruanos, 95 poseen por lo menos algún tipo de TIC; además, el acceso a estas depende del grado de instrucción logrado por el jefe de la familia; es decir, aquellos con educación superior demuestran un acceso de 99.6\%. Por otro lado, acerca de la posesión de computadoras, son los hogares de Lima Metropolitana los que encabezan la lista con $52.9 \%$, mientras que, el Resto urbano y los hogares rurales tienen una posesión de 38.3 y $7.5 \%$ respectivamente; acerca del acceso a Internet el 62.9\% de Lima Metropolitana tiene el servicio en su hogar, el 40.5 en el Resto urbano y el 5.9\% en los hogares rurales, aunque mayormente lo usan a través de los móviles (Inei, 2020).

Estos datos reflejan que la brecha de acceso, en el Perú, se ha reducido considerablemente en la ciudad, lo cual favorece a la implementación de la modalidad virtual en la educación superior; sin embargo, el gran reto está en la zona rural del país. Además, si bien el índice de tenencia de Internet ha aumentado a la fecha, ello no ha implicado que el índice de su uso adecuado también lo haya hecho.

Una importante iniciativa acerca de esta última brecha fue cuando, en el 2007, a través del programa "Maestros Siglo XXI: Una laptop para cada docente", el gobierno otorgó bonos a los profesores nombrados en escuelas públicas para la adquisición de laptops (Cuenca et al., 2009), beneficio que fue complementado con un préstamo a cuatro años de plazo por parte del Banco de la Nación.

Sin embargo, el poseer una laptop y desarrollar competencias digitales debe ser complementado con una conectividad de alta velocidad de acceso libre en los campus universitarios y lugares de investigación. Por ejemplo, Chile aplicó, para el sector escolar, el proyecto denominado Conectividad para la Educación 2030 y pretende extender el servicio a más de diez mil instituciones educativas en un plazo máximo de diez años
(Sistema de información de tendencias educativas en América Latina, 2020).

En cuanto a la brecha de tercer orden, aquella que aborda la problemática de la carencia o desigualdad de competencias digitales tanto en docentes como en estudiantes, el Minedu creó en el 2001 una plataforma educativa hoy llamada PerúEduca con el objetivo de transferir conocimientos a toda la comunidad educativa, $\mathrm{y}$, hasta el 2016, dicha plataforma ya contaba con más de 6,388 recursos educativos digitales para descargar libremente (Minedu, 2016b). Dichos datos resultan alentadores para el nivel educativo de la Educación Básica; sin embargo, es necesario considerar el planteamiento de nuevos criterios e instrumentos pertinentes que midan y promuevan el desarrollo de la competencia digital de los docentes en el nivel de la Educación Superior Universitaria.

En el 2016, la versión del Currículo Nacional de Educación Básica incluyó por primera vez el desarrollo de la competencia digital para los estudiantes, a través de la competencia 28 , que indica "se desenvuelve en los entornos virtuales generados por las TIC" (Ministerio de Educación [Minedu], 2016a, p.84). Asimismo, respecto de los docentes, se incluyó en el Diseño Curricular Básico de la Formación Inicial Docente del nivel Inicial, Primaria e Intercultural Bilingüe del 2019 la competencia "gestiona los entornos digitales para su desarrollo profesional y práctica pedagógica"; además, PerúEduca ya ha registrado más de 5,000 docentes que han culminado el curso de alfabetización digital a nivel nacional (Minedu, 2019).

\section{Las Competencias Digitales}

La competencia digital es entendida como la sumatoria de una serie de habilidades, conocimientos y actitudes en un contexto tecnológico e informático (Gisbert \& Esteve, 2011) que, en la actualidad, ya no son expectativas que correspondan exclusivamente a ciertos grupos privilegiados de la sociedad pues a partir de la globalización, estas se han convertido en uno de los logros esenciales (Instituto Nacional de Tecnologías Educativas y de Formación del profesorado [Intef], 2017; Gisbert \& Esteve, 2011) que todo estudiante al egresar de la educación básica debe 
haber alcanzado para poder insertarse en la vida adulta y responder, con éxito, las exigencias que esta demanda. Estas competencias adquiridas no solo le garantizarán la capacidad de aprovechar los beneficios de las nuevas tecnologías digitales y sus retos, sino que también lo harán partícipe de la nueva sociedad del conocimiento (Intef, 2017; Gisbert \& Esteve, 2011).

Ante este horizonte, es sobre los docentes en quienes recae la responsabilidad de asegurar, en los estudiantes, el desarrollo de dichas nuevas competencias. Es por ello que son los docentes quienes primero deben adquirir la capacidad para convertirse en guías acordes con los nuevos medios y con el manejo del tipo de conocimiento y habilidades pertinentes, sobre todo aquellas relacionadas con la gestión del conocimiento (Intef, 2017). El desarrollo de las competencias en mención debe convertirse en prioridad dentro de sus áreas o especialidades. Por ello, surge la necesidad de poner mucha atención en los sistemas de formación de profesorado y el reconocimiento de su desarrollo profesional (Intef, 2017; Rangel \& Peñalosa, 2013).

En el 2008, la Unesco estableció tres aspectos o dimensiones sobre competencias digitales docentes: 1) Comprensión e integración de competencias tecnológicas; 2) Resolución de problemas concretos, aplicando conocimientos tecnológicos y 3) Producción de nuevos conocimientos a partir de los ya generados. Por ello, el desarrollo de competencias digitales docentes que los haga capaces de diseñar y administrar programas de la misma naturaleza debe ser parte de la implementación de las nuevas políticas de formación e-learning. Estas competencias deben estar enfocadas en el diseño de estrategias para la enseñanza y el aprendizaje, tales como el trabajo colaborativo, la difusión del hábito de la lectura y la investigación, el desarrollo de la autonomía en los estudiantes y el diseño de nuevos métodos de evaluación (Yong et al., 2017).

Asimismo, Durán et al. (2016) realizaron un análisis comparativo de los diferentes modelos de competencia digital con el objetivo de obtener un estándar para el docente universitario fundamentado en tres dimensiones: la básica, que implica el desenvolvimiento en el plano tecnológico, comu- nicativo, informacional, multimedia, entre otros; específicas, como la gestión y la evaluación de los aprendizajes apoyados en el uso de las TIC, así como la identificación del potencial didáctico de estas, su implicancia en la formación, concreción de los aprendizajes y desarrollo de la creatividad de los estudiantes; $\mathrm{y}$, concretas, relacionadas con la investigación e innovación a partir del uso de las TIC y la promoción del uso de estas generando publicaciones y difundiéndolas en la red de Internet.

Además, García-Quismondo y Cruz-Palacios (2018) señalan que las competencias digitales en el sector educativo son instrumentos imprescindibles que promueven el desarrollo de habilidades y nuevas competencias vinculadas con la transferencia de conocimientos e innovación. También, Iordache et al. (2017) plantean que estas deben considerarse como logros de aprendizaje medibles en cuanto a la alfabetización digital se refiere. Por otro lado, las competencias digitales deben integrar, como un todo, conocimientos y capacidades de índole tecnológico, cuya enseñanza y aprendizaje deben desarrollarse, con carácter de prioridad, en la educación superior y a través de la implementación de herramientas de alta complejidad tecnológica, pero con fines didácticos (Ocaña-Fernández et al., 2019).

Respecto de los docentes, las nuevas políticas educacionales referidas a las competencias digitales deben estar orientadas a una clara exigencia del empleo de las TIC y una actitud favorable ante estas, el desarrollo de habilidades en técnicas informáticas (manejo instrumental), constante capacitación y gestión del conocimiento (Rangel \& Peñalosa, 2013).

En cuanto a los estudiantes, uno de los objetivos de las nuevas políticas educacionales para la modalidad e-learning debe ser el diseño de estrategias de aprendizaje estandarizadas que garanticen la adquisición de la competencia digital del alumnado universitario (Gisbert \& Esteve, 2011) con la finalidad de conseguir los objetivos educativos de formación universitaria; $y$, en este camino, el diseño de la evaluación pertinente que obedezca a la metodología usada en dicha modalidad (Mora-Vicarioli, 2019). Por lo tanto, el proceso de evaluación final de la competencia digital en los estudiantes se debe agilizar y operativizar 
en cada nivel educativo, pues la adquisición de dichas competencias no solo asegura una buena estancia universitaria, sino que prepara para las exigencias que demanda el mundo laboral (Gisbert \& Esteve, 2011).

Finalmente, para Rodríguez (2015) la importancia de estas competencias radica en que son capaces de motivar al estudiante y al docente en la innovación en las TIC; asimismo, el docente asume nuevos roles para los cuales necesita de una formación psicopedagógica, tecnológica sustentada en la actualización profesional, permite también la transformación de la información en conocimiento crítico; mientras que, al estudiante le promueve competencias de autogestión (aprender a aprender).

\section{Evaluación en la Modalidad E-learning}

Las políticas empleadas, para el desarrollo de la calidad de la modalidad e-learning por parte de las instituciones, deben brindar apoyo a los agentes educativos y a los docentes recién incorporados; asimismo, deben diseñar cursos de calidad con adecuada infraestructura tecnológica, donde el proceso de evaluación al estudiante se desarrolle sobre la base de la autenticación de este, la autoría de su trabajo y un examen de seguridad; así como adecuadas medidas de seguridad electrónica y acceso a la educación para estudiantes con discapacidad, enfermedad o circunstancias atenuantes (Huertas et al., 2018).

Complementan Huertas et al. (2018) que las instituciones educativas deben procurar que su enseñanza anime a los estudiantes a protagonizar su propio proceso de aprendizaje (autonomía); en ese sentido, la evaluación debe reflejar esta tendencia. Sin embargo, la flexibilidad y autonomía en esta modalidad podría implicar también un riesgo de abandono de los cursos debido a la percepción de soledad. Por esto, hay que tener en cuenta la gestión del tiempo (García-Peñalvo, $2020 b$ ) con estrategias adecuadas de comunicación hacia los estudiantes para promover su respectiva adaptación a la modalidad. Aquí, las herramientas síncronas (tiempo real) como espacios de interacción entre el docente y sus estudiantes podrían jugar un rol importante, pues facilitan la realimentación oportuna (Mora-Vicarioli, 2019).

La evaluación debe estar orientada a tomar como punto de partida los conocimientos previos del estudiante (evaluación diagnóstica) para que el docente pueda efectuar cambios en función de las necesidades de este, con lo cual se reafirma la importancia de la flexibilidad en la modalidad e-learning que permite los ajustes en contenidos y actividades sobre la marcha (Mora-Vicarioli, 2019). Asimismo, esta modalidad facilita la evaluación continua y el papel protagónico del estudiante en las actividades colaborativas (Fardoun et al., 2020) que movilizan y desarrollan, a su vez, las habilidades blandas (Mora-Vicarioli, 2019; García-Peñalvo, 2020b).

Por último, es imprescindible pensar que la evaluación del aprendizaje, en un entorno virtual, debe ser rediseñada para alejarla de la sombra presencial; por tanto, requiere del análisis de una serie de aspectos como el nivel educativo alcanzado, la ubicación geográfica de los estudiantes a quienes va dirigida, la actitud de los involucrados frente al uso de TIC, la tecnología a emplear, la metodología y la gestión (Fardoun et al., 2020).

\section{Desafíos}

Es importante asumir que el proceso de migración de estrategias y metodologías de lo presencial a lo virtual no ocurrirá aceleradamente, pues se deben superar brechas muy importantes antes (Sterzer, 2020).

Ello implica una reforma total del sistema de cada institución, pues no se trata solo de migrar de una plataforma a otra, como aseguran algunos que ha ocurrido en este tiempo de pandemia acuñando el término "Coronateaching" (Ramos, 2020), sino de transformar cada instancia relacionada con el servicio educativo que se brinda para que cada una apunte hacia un mismo norte (García-Peñalvo, 2020b). Por esto, es importante considerar que cada nivel educativo ofrece, al sector universitario, una variada gama de experiencias, pues los estudiantes pese a pertenecer a un mismo grupo presentan diferencias marcadas en cuanto a la autonomía para el aprendizaje, el nivel de conocimientos y el dominio de ciertas habilidades (Sánchez et al., 2020). Asimismo, los docentes son una pieza clave en este proceso de transición, a partir de que se les forme en metodologías digitales (Hurtado, 2020). 
Asimismo, es necesario considerar cierta flexibilidad en cuanto al proceso de evaluación de la calidad del servicio en las universidades. Por esto, Alcántara (2020) propone la implementación de iniciativas para el desarrollo de capacidades con el fin de facilitar la transición al aprendizaje en línea.

Al respecto, García-Peñalvo (2020b) propone un modelo de referencia para la educación no presencial, en el cual plantea los siguientes elementos: identidad, rasgos propios de las instituciones presenciales materializados en el diseño de imagen, un portal web y los medios sociales; la estructura tecnológica (implica gestión, infraestructura física y lógica); servicios de apoyo (confidencialidad, análisis de información académica, atención al usuario); contenidos, los cuales, por sí mismos, no conducen al éxito académico (García-Peñalvo, 2020b; Huertas et al., 2018), por el contrario requieren de gestiones por parte de los especialistas, actualizaciones, reconocimiento de autoría y complementos con medio multimedia que deben ser evaluados de forma continua o final; modelo pedagógico, el cual es la suma del diseño de las acciones formativas, contenidos, del servicio y la tecnología. Todos estos servicios académicos deben obedecer, en su totalidad, a la naturaleza de la modalidad, política y estrategia orientada a un verdadero proyecto institucional que conduzca a una transformación digital de la institución e involucre a todos sus miembros (García-Peñalvo, 2020b).

Por lo tanto, el potenciar el trabajo colaborativo entre docentes; replantear el logro de aprendizaje y la forma de evaluar a los estudiante;s y adoptar la modalidad blended como estrategia, son tres de los principales desafíos que se deben tomar en cuenta para el diseño y la gestión de las aulas virtuales en la educación superior (De Vincenzi, 2020).

Por otro lado, en el proceso de transición de las universidades a la modalidad e-learning, Alcántara (2020) sostiene que los gobiernos deberían proveer de financiamiento (préstamos) a los ciudadanos en etapa de estudios o de enseñanza a modo de estímulo, medida que debe estar ligada a la libre conectividad en lugares de estudio e investigación.

Además, resaltar la importancia del aporte que pueden brindar las universidades u otras entida- des de educación superior en las diferentes áreas profesionales para que estén involucradas y actualizadas en cuanto al uso de las TIC a través de capacitaciones (Grupo Banco Mundial, 2020). En cuanto a la lucha por la equidad del servicio en la educación superior y porque este sea accesible para todos, la universidad debe ocupar un papel importante y asumir la responsabilidad que le corresponde frente a las necesidades sociales ( $\mathrm{Gu}$, 2020). Ello obliga a analizar su función en la sociedad y su contribución a la búsqueda de soluciones a los problemas del contexto de pandemia y pospandemia.

Por esto, la creación de una alianza estratégica entre universidades para compartir buenas prácticas en educación virtual se perfila como una respuesta conveniente. Al respecto, Chile ha establecido puentes de conexión entre universidades públicas y privadas para compartir buenas prácticas y promover la capacitación docente a aquellas instituciones no participantes de esta alianza, esto logrado con la mediación de su respectivo Ministerio de Educación (Organización de las Naciones Unidas para la Educación, la Ciencia y la Cultura \& Instituto Internacional para la Educación Superior en América Latina y el Caribe [Unesco-Iesalc], 2020). Asimismo, sería pertinente pensar en el establecimiento de comisiones de educación superior virtual que puedan orientar y absolver dudas en el funcionamiento (apoyo tecnológico en plataformas virtuales) y recomendar metodologías ya usadas en la enseñanza y aprendizaje en esta modalidad (efecto multiplicador de la alianza estratégica).

En cuanto al reto de la alfabetización digital, tanto para docentes como para los estudiantes de educación universitaria, este es de alta prioridad. Aunque la necesidad de dichas competencias siempre se mantuvo latente, ahora, frente al contexto de pandemia, urge implementarlas (Sterzer, 2020). Por ello, surge la necesidad de formar a los estudiantes en competencias digitales desde la cantera (educación básica) para que esto impacte en la educación superior. Uruguay y Argentina dan muestra de ello a través de políticas digitales nacionales: Plan Ceibal y Educ.ar respectivamente (Sistema de información de tendencias educativas en América Latina, 2020). Dichas iniciativas 
han logrado respuestas oportunas ante la crisis sanitaria por la COVID-19.

Para García-Peñalvo (2020b), la educación virtual podría mejorar considerablemente si se define el tamaño grupal de estudiantes (de $30 \mathrm{a}$ 40), se define el tiempo de respuesta del docente sobre las actividades asíncronas, se establece una carga realista de actividades por semana, se respeta las autorías y licencias de materiales, se evalúa según perfil de estudiantes y se acredita a los docentes en tecnología para la enseñanza.

Sin embargo, antes de inclinarse por algún mecanismo acertado capaz de mejorar el ejercicio del e-learning, Pedró (2020) manifiesta que es preciso que las instituciones universitarias registren los cambios pedagógicos empleados durante la pandemia, de esta manera, fomenten la reflexión interna sobre sus propios modelos de enseñanza y aprendizaje para aprender de los errores; este camino permitirá un verdadero escalamiento a la digitalización.

\section{Discusión}

A partir de lo hallado, se puede asegurar que es de suma importancia la consideración de las tres brechas: de acceso, de uso y competencial que Fernández (2020), García-Peñalvo (2020) y Pedró (2020) en una suerte de complemento han identificado dentro del contexto educativo en tiempos de pandemia; de esta manera, es preciso establecer nuevas condiciones básicas de calidad del servicio educativo e-learning en el nivel superior universitario, ya que las existentes se elaboraron sobre la base de una educación presencial con un mínimo porcentaje de virtualidad, tal como lo establece el artículo 47 de la Nueva Ley Universitaria, Ley $\mathrm{N}^{\circ} 30220$ (2014).

De esta manera, la adopción de modalidades alternativas fundamentadas en la virtualidad dependerá de las instituciones universitarias, así como su adaptación a las exigencias del nuevo mercado educativo y a los perfiles de sus potenciales estudiantes. En consecuencia, dichos cambios deben traer consigo, no solo la innovación y la adaptación del servicio educativo a los nuevos contextos, sino, también, la mejora de la calidad del servicio para erradicar aquellas ideas orientadas al cuestionamiento del mismo (Figallo et al., 2020).

La ampliación de la oferta educativa e-learning se sustenta en algunos beneficios que genera en el estudiante como potencialidades comunicativas (interacción y colaboración) sujetas al papel docente y las estrategias didácticas (Fardoun et al., 2020; Mora-Vicarioli, 2019). Sin embargo, el buen desempeño en este entorno, requiere que los agentes educativos posean y empleen competencias digitales

Sin embargo, ante las deficiencias en competencias digitales de los agentes educativos para asumir adecuadamente el nuevo contexto virtual, es imprescindible y urgente la implementación de programas de alfabetización digital mucho más intensivos, así como un cambio personal y radical de paradigma acerca de la educación actual. Han transcurrido casi veinte años desde la creación del Proyecto Huascarán, del portal PerúEduca, de la entrega de bonos y préstamos para la adquisición de laptops y de la compra de laptops $\mathrm{XO}$, por mencionar algunas iniciativas; sin embargo, hasta el momento más de uno de los mencionados hoy es parte de los intentos fallidos en la inserción en la alfabetización digital.

En cuanto a la intervención del gobierno, Alcántara (2020) sugiere su participación a través de programas que contribuyan con el acceso a la tecnología por medio de préstamos gubernamentales a los ciudadanos relacionados con la educación. Al respecto, como apuntamos en páginas anteriores, en el 2007 el gobierno peruano otorgó un bono para que docentes nombrados puedan adquirir una laptop (Cuenca et al., 2009). Es aquí cuando el gobierno desaprovechó una valiosa oportunidad de iniciar con fuerza el fomento para el desarrollo de la alfabetización digital.

A lo anterior, se suma la necesidad de conectividad de alta velocidad que debería ser de acceso libre en los campus universitarios y lugares de enseñanza e investigación, tal como lo hizo Chile para el sector escolar (Sistema de información de tendencias educativas en América Latina, 2020).

Además, puede fomentar alianzas estratégicas entre universidades para compartir buenas prácticas en educación virtual, como lo ha hecho Chi- 
le (Unesco-Iesalc, 2020). Asimismo, un complemento a dichas alianzas sería el establecimiento de comisiones de educación superior virtual que puedan orientar y absolver dudas en el funcionamiento (apoyo tecnológico en plataformas virtuales) y recomendar metodologías ya usadas en la enseñanza y aprendizaje en esta modalidad (efecto multiplicador de la alianza estratégica).

Asimismo, debe dar mayor impulso a la formación en competencias digitales desde la educación básica, un buen comienzo es la competencia 28 del Diseño Curricular Nacional (Minedu, 2016a); sin embargo, se requiere de más acciones, esto para impactar positivamente en la educación superior. Al respecto, tanto Uruguay como Argentina dan sendas muestras de verdaderas políticas digitales en sus ámbitos nacionales a través del Plan Ceibal y Educ.ar (Sistema de información de tendencias educativas en América Latina, 2020), respectivamente, las cuales han logrado respuestas oportunas ante la crisis sanitaria por la COVID-19.

El diseño de nuevos instrumentos y estrategias de enseñanza y evaluación estandarizados, la gestión e implementación de sesiones online, el trabajo colaborativo, entre otros, deben ser las principales competencias para desarrollar en los docentes, sin dejar de lado el aspecto técnico, como lo es el manejo y conocimiento de una gama de herramientas digitales para la modalidad e-learning. Todo ello debe apuntar a la implicancia de una política educacional que construya el nuevo perfil del docente del nivel superior universitario, puesto que este es uno de los ejes fundamentales para que el proceso de transición del proceso enseñanza-aprendizaje se lleve a cabo con éxito.

En cuanto a los estudiantes, es deber de las universidades estar a la vanguardia con respecto al desarrollo de las competencias digitales de su población estudiantil. Sin embargo, para que ello se concrete, las nuevas políticas educacionales para la enseñanza e-learning deben establecer la implementación de estándares de evaluación que midan el desarrollo de dichas competencias, así como de herramientas de enseñanza pertinentes con las condiciones básicas de calidad.

Además, los especialistas coinciden en que la calidad de esta estaría sostenida en la medida en que las instituciones procuren orientarse a las siguientes propuestas: en cuanto a los estudiantes, la cantidad del tamaño grupal no debe exceder de 40; la carga semanal de actividades debe planificarse de acuerdo a la realidad de cada grupo y la evaluación debe plantearse en función de la diversidad, competencias del curso, el grado de presencialidad, y la identificación del estudiante (Huertas et al., 2018); y, el control de su entorno. En cuanto a los docentes, estos deben estar acreditados y en continua formación respecto de las tecnologías para la enseñanza, modelos pedagógicos e innovaciones pedagógicas; manejar herramientas para detectar el plagio y cuidar el uso correcto de autorías, y la retroalimentación que brindan a sus estudiantes debe estar enmarcada en un tiempo máximo de respuesta para poder alcanzar el logro del curso (García-Peñalvo, 2020b).

Finalmente, cabe resaltar que, antes de tomar en cuenta dichas propuestas, será de suma importancia dirigir la mirada hacia la experiencia generada durante la pandemia, recoger las estrategias pedagógicas aplicadas durante esta, tomando en cuenta aciertos y errores; y fomentar la reflexión, en cada institución, en cuanto a sus modelos de enseñanza y aprendizaje para poder acceder a una verdadera transformación digital (Pedró, 2020).

\section{Referencias}

Alcántara, A. (2020). Educación superior y COVID-19: una perspectiva comparada. En H. Casanova (Coord.), Educación y pandemia: una visión académica (pp. 75-82). Universidad Nacional Autónoma de México, Instituto de Investigaciones sobre la Universidad y la Educación. http://132.248.192.241:8080/jspui/ bitstream/IISUE_UNAM/541/1/AlcantaraA_2020_ Educacion_superior_y_covid.pdf

Buendía, A. (2020). Desafíos de la educación superior en tiempos de pandemia: la contingencia inesperada. REPORTE CESOP, 132, 25-32. https://www.researchgate.net/profile/Abigail-Rodriguez-Nava/ publication/346642075_COVID-19_La_humanidad_a_prueba/links/5fd52c1445851553aOb15466/COVID-19-La-humanidad-a-prueba.pdf\#page=26

Cevallos, M. \& Caraguay, J. (2013). Tecnología informática aplicada a la educación a distancia. Aplicativo: prototipo de aula virtual [Tesis de licenciatura]. Universidad Técnica del Norte. http://repositorio.utn. 
edu.ec/handle/123456789/1113

Cuenca, R., Ames, P., Córdova, S., Guerrero, L., Iguiñiz, M. Muñoz, F., Rivero, J., Tovar, T., Trapnell, L., \& Zavala, V. (2009). La educación en los tiempos del APRA: Balance 2006 - 2009. Foro Educativo.

Decreto Legislativo $N^{\circ} 1496$ de 2020 [con fuerza de ley]. Por medio del cual se establece disposiciones en materia de educación superior universitaria en el marco del estado de emergencia sanitaria a nivel nacional. 10 de mayo de 2020. D.O. $\mathrm{N}^{\circ} 15413$.

Durán, M., Gutiérrez, I., \& Prendes, M. (2016). Análisis conceptual de modelos de competencia digital del profesorado universitario. RELATEC: Revista Latinoamericana de Tecnología Educativa, 15(1), 97-114. https://doi.org/10.17398/1695-288X.15.1.97

Fardoun, H., González, C, Collazos, C., \& Yousef, M. (2020). Estudio exploratorio en Iberoamérica sobre procesos de enseñanza-aprendizaje y propuesta de evaluación en tiempos de pandemia. Education in the Knowledge Society, 21, 1-9. https://doi.org/10.14201/ eks. 23437

Fernández, M. (2020). Una pandemia imprevisible ha traído la brecha previsible. Cuaderno de campo. chttps:// blog.enguita.info/2020/03/una-pandemia-imprevisible-ha-traido-la.html

Figallo, F, González, M.T., \& Diestra, V. (2020). Perú: educación superior en el contexto de la pandemia por el Covid-19. ESAL-Revista de Educación Superior en América Latina, 20-28. https://rcientificas.uninorte.edu.co/index.php/esal/article/ view/13404/214421444832

García-Quismondo, M. A., \& Cruz-Palacios, E. (2018). Gaming como Instrumento Educativo para una Educación en Competencias Digitales desde los Academic Skills Centres. Revista General de Información y Documentación, 28(2), 489-506. https://doi. org/10.5209/RGID.62836

García-Peñalvo, F. J. (2020a). El sistema universitario ante la COVID-19: corto, medio y largo plazo. Universídad. https://www.universidadsi.es/sistema-universitario-covid-19/

García-Peñalvo, F. J. (2020b). Modelo de referencia para la enseñanza no presencial en universidades presenciales. Campus Virtuales, 9(1), 41-56.

Gisbert, M., \& Esteve, F. (2011). Digital Leaners: la competencia digital de los estudiantes universitarios. La cuestión universitaria, 7, 48-59. http://polired. upm.es/index.php/lacuestionuniversitaria/article/ view/3359/3423

Gortazar, L., Moreno, J.M., \& Zubillaga, A. (2020). Informe covid-19 y Educación II: escuela en casa y desigualdad. https://www.researchgate.net/publication/341434688_INFORME_COVID19_Y_EDUCACION_II_Escuela_en_casa_y_desigualdad/link/5ec03f8ca6fdcc90d67a62d1/download

Grupo Banco Mundial. (2020). Covid-19: impacto en la educación y respuestas de política pública. http://documents1.worldbank.org/curated/en/ 804001590734163932/pdf/The-COVID-19-Pandemic-Shocks-to-Education-and-Policy-Responses.pdf

Gu, R. (2020). Reflexiones sobre el modelo universitario visto desde la crisis del coronavirus. Reflexiones. Revista Facultad de Ciencias Sociales. Universidad de Costa Rica, 99(2), 1-10. https://www.doi.org: 10.15517/ rr.v99i2.42151

Huertas, E., Biscan, I., Ejsing, C., Kerber, L., Kozlowska L., Marcos, S., Lauri, L., Risse, M., Schorg, K., \& Seppmann, G. (2018). Considerations for quality assurance of e-learning provision. Report from the ENQA working group VIII on quality assurance and e-learning. https://www.enqa.eu/wp-content/ uploads/Considerations-for-QA-of-e-learning-provision.pdf

Hurtado, F. (2020). La educación en tiempos de pandemia: los desafíos de la escuela del siglo XXI. CIEG, Revista arbitrada del centro de investigación y estudios gerenciales, 44, 176-187. http://www.grupocieg.org/ archivos_revista/Ed.44(176-187)\%20Hurtado\%20Tavalera_articulo_id650.pdf

Instituto Nacional de Estadística e Informática (2020). Es tadísticas de las Tecnologías de Información y Comunicación en los Hogares. https://www.inei.gob. pe/media/MenuRecursivo/boletines/boletin_tics. pdf

Instituto Nacional de Tecnologías Educativas y de Formación del profesorado (2017). Marco Común de Competencia Digital Docente. http://educalab.es/ documents/10180/12809/Marco+competencia+digital+docente+2017/afb07987-1ad6-4b2d-bdc858e9faeeccea

Iordache, C., Mariën, I., \& Baelden, D. (2017). Developing Digital Skills and Competences: A QuickScan Analysis of 13 Digital Literacy Models. Italian Journal of Sociology of Education, 9(1), 6-30. https://doi. org/10.14658/pupj-ijse-2017-1-2

Ley $\mathrm{N}^{\circ}$ 30220. Ley Universitaria. (3 de Julio de 2014). https://www.google.com/search? q=ley+uni versitaria $+30220+e l+$ peruano + pdf $\&$ rlz $=1 C$ 1CHBD_esPE906PE906\&oq=ley+univer\&aqs=chrome.0.69i59j69i57j69i59l2j69i60l3j69i65.3373j0j7\&sourceid $=$ chrome $\&$ ie $=U T F-8 \#$

Ministerio de Educación (2016a). Currículo Nacional de Educación Básica. http://www.minedu.gob.pe/curriculo/pdf/curriculo-nacional-2016-2.pdf

Ministerio de Educación (2016b). Portal PerúEduca del Minedu cumple 15 años al servicio de la comunidad educativa del país. http://www.minedu.gob.pe/n/ noticia.php?id=40701

Ministerio de Educación (2019). Educación para un mundo digital. https://www.gob.pe/institucion/minedu/ informes-publicaciones/345597-educacion-para-un-mundo-digital

Mora-Vicarioli, F. (2019). Estado del arte de la evaluación de los aprendizajes en la modalidad del e-learning 
desde la perspectiva de evaluar para aprender: precisiones conceptuales. Revista Electrónica Calidad en la Educación Superior, 10(1), 58-95. https://revistas.uned.ac.cr/index.php/revistacalidad/article/ view/2453

Ocaña-Fernández, Y., Valenzuela-Fernández, L., \& Garro-Aburto, L. (2019). Inteligencia artificial y sus implicaciones en la educación superior. Propósitos y Representaciones, 7(2), 536-568. http://dx.doi. org/10.20511/pyr2019.v7n2.274

Organización de las Naciones Unidas para la Educación, la Ciencia y la Cultura (2008). Estándares de competencia en TIC para docentes. https://eduteka.icesi. edu.co/pdfdir/UNESCOEstandaresDocentes.pdf

Organización de las Naciones Unidas para la Educación, la Ciencia y la Cultura (2020a). Crisis y currículo durante el COVID-19: Mantención de los resultados de calidad en el contexto del aprendizaje remoto. https://unesdoc.unesco.org/ark:/48223/ pf0000373273_spa

Organización de las Naciones Unidas para la Educación la Ciencia y la Cultura (2020b). ¿Qué precio pagará la educación por COVID-19? http://www.iiep. unesco.org/en/what-price-will-education-pay-covid-19-13366

Organización de las Naciones Unidas para la Educación, la Ciencia y la Cultura \& Instituto Internacional para la Educación Superior en América Latina y el Caribe (2020). COVID-19 y educación superior: De los efectos inmediatos al día después. http://www.iesalc. unesco.org/wp-content/uploads/2020/05/COVID19-ES-130520.pdf

Pedró, F. (2020). COVID-19 y educación superior en América Latina y el Caribe: efectos, impactos y recomendaciones políticas. Análisis Carolina. https://www.fundacioncarolina.es/wp-content/uploads/2020/06/ AC-36.-2020.pdf

Ramos, D. (2020). Coronateaching isíndrome o nueva oportunidad para la reflexión? I/II. Unesco. https://www. iesalc.unesco.org/2020/07/02/coronateaching-sindrome-o-nueva-oportunidad-para-la-reflexion-i-ii/

Rangel, A., \& Peñalosa, E. (2013). Alfabetización digital en docentes de educación: construcción y prueba empírica de instrumento de evaluación. Píxel-Bit. Revista de Medios y Educación, 43, 9-23. https://recyt. fecyt.es/index.php/pixel/article/view/61545

Resolución del Consejo Directivo N. ${ }^{\circ}$ 039-2020-SUNEDU-CD (2020). https://www.sunedu.gob.pe/resolucion-del-consejo-directivo-n-039-2020-sunedu-cd/

Rodríguez, I. (2015). La importancia de las competencias digitales de los docentes, en la sociedad del conocimiento. Revista Iberoamericana de Producción Académica y Gestión Educativa, 2, 1-12. https://pag. org.mx/index.php/PAG/article/view/484/523

Sánchez, M., Martínez, A.M., Torres, R., De Agüero, M., Hernández, A.K., Benavides, M.A., Rendón, V.J., \& Jaimes, C.A. (2020). Retos educativos durante la pandemia de COVID-19: una encuesta a profesores de la UNAM. Revista Digital Universitaria , 21(3), 2-24. https://www.revista.unam.mx/wp-content/uploads/ a12.pdf

Sistema de información de tendencias educativas en América Latina (2020). Sistematización de respuestas de los sistemas educativos de América Latina a la crisis de la COVID-19. https://www.siteal.iiep.unesco.org/ respuestas_educativas_covid_19

Sterzer, S. (2020). Impacto del coronavirus en el sistema educativo: ejemplos en el continente asiático. Revista electrónica del Departamento de Ciencias Sociales, 7(2), 64-74. https://ri.unlu.edu.ar/ xmlui/bitstream/handle/rediunlu/751/10.-Impac to-del-coronavirus-en-el-sistema-educativo_-ejem plos-en-el-continente-asi\%c3\%a1tico..pdf?sequence $=1$ \&isAllowed $=\mathrm{y}$

Tito, M.D., \& Serrano, B. (2016). Desarrollo de soft skills una alternativa a la escasez de talento humano. INNOVA Research Journal, 1(12), 59-76. http://201.159.222.115/ index.php/innova/article/view/81/142

De Vincenzi, A. (2020). Del aula presencial al aula virtual universitaria en contexto de pandemia de COVID-19. Universidad Abierta Interamericana. https://uai. edu.ar/media/115153/4-del-aula-presencial-al-aula-virtual-universitaria-en-contexto-de-pandemia-covid-19.pdf

Yong, E., Nagles, N., Mejía, C., \& Chaparro, C. (2017). Evolución de la educación superior a distancia: desafíos y oportunidades para su gestión. Revista Virtual Universidad Católica del Norte, 50, 81-105. https://www. redalyc.org/pdf/1942/194250865006.pdf

RIDU / Revista Digital de Investigación en Docencia Universitaria / e-ISSN: 2223-2516

(c) Los autores. Este artículo es publicado por la Revista Digital de Investigación en Docencia Universitaria del Área de Institutional Research and Effectiveness de la Dirección de Aseguramiento de la Calidad, Universidad Peruana de Ciencias Aplicadas. . "Este es un artículo de acceso abierto, distribuido bajo los términos de la LicenciaCreativeCommons Atribución 4.0 Internacional (CC BY 4.0) (https://creativecommons.org/licenses/by/4.0/deed.es), que permite el uso, distribución y reproducción en cualquier medio, siempre que la obra original sea debidamente citada." 